\title{
Lead Placement Standard Intercostal Space Higher
}

National Cancer Institute

\section{Source}

National Cancer Institute. Lead Placement Standard Intercostal Space Higher. NCI

Thesaurus. Code C71117.

An electrocardiographic lead placement schema in which the "V" leads are placed one intercostal space cephalad to the position they would have in the standard lead placement schema. 